\title{
THE DIFFERENCE BETWEEN THE PRODUCT AND THE CONVOLUTION PRODUCT OF DISTRIBUTION FUNCTIONS IN $\mathbb{R}^{n}$
}

\author{
E. Omey and R. Vesilo
}

\author{
Communicated by Slobodanka Janković
}

\begin{abstract}
Assume that $\vec{X}$ and $\vec{Y}$ are independent, nonnegative $d$-dimensional random vectors with distribution function (d.f.) $F(\vec{x})$ and $G(\vec{x})$, respectively. We are interested in estimates for the difference between the product and the convolution product of $F$ and $G$, i.e.,

$$
D(\vec{x})=F(\vec{x}) G(\vec{x})-F * G(\vec{x}) .
$$

Related to $D(\vec{x})$ is the difference $R(\vec{x})$ between the tail of the convolution and the sum of the tails:

$$
R(\vec{x})=(1-F * G(\vec{x}))-(1-F(\vec{x})+1-G(\vec{x})) .
$$

We obtain asymptotic inequalities and asymptotic equalities for $D(\vec{x})$ and $R(\vec{x})$. The results are multivariate analogues of univariate results obtained by several authors before.
\end{abstract}

\section{Introduction}

Assume that $\vec{X}$ and $\vec{Y}$ are independent, nonnegative $d$-dimensional random vectors with distribution function (d.f.) $F(\vec{x})$ and $G(\vec{x})$, respectively. In this paper, we are interested in estimates for the difference between the product and the convolution product of $F$ and $G$, i.e.,

$$
D(\vec{x})=F(\vec{x}) G(\vec{x})-F * G(\vec{x}) .
$$

Here, and throughout the paper, we set

$$
F * G(\vec{x})=P(\vec{X}+\vec{Y} \leqslant \vec{x})=\int_{\overrightarrow{0}}^{\vec{x}} F(\vec{x}-\vec{y}) d G(\vec{y}) .
$$

2010 Mathematics Subject Classification: Primary 26A12, 26B99, 60E99, 60K99.

Key words and phrases: Subexponential distribution, regular variation, $O$-regularly varying functions, sums of random vectors. 
Clearly, we have $D(\vec{x}) \geqslant 0$. Related to $D(\vec{x})$ is the difference between the tail of the convolution and the sum of the tails:

$$
R(\vec{x})=\overline{F * G}(\vec{x})-\bar{F}(\vec{x})+\bar{G}(\vec{x}),
$$

where, here and elsewhere, we use the notation $\bar{F}=1-F$ for the tails of d.fs. Clearly, we have

$$
R(\vec{x})=D(\vec{x})-\bar{F}(\vec{x}) \bar{G}(\vec{x}) .
$$

In the case of one single d.f. $F$, we are interested in the following differences:

$$
D_{n}(\vec{x})=F^{n}(\vec{x})-F^{* n}(\vec{x}) \text {, and } R_{n}(\vec{x})=\overline{F^{* n}}(\vec{x})-n \bar{F}(\vec{x}) \text {. }
$$

Note that we have

$$
\left|R_{n}(\vec{x})-D_{n}(\vec{x})\right| \leqslant(1-F(\vec{x}))^{2} \frac{n(n-1)}{2} .
$$

The interest in $D_{n}$ and $R_{n}$ comes from the class $S\left(\mathbb{R}^{d}\right)$ of subexponential distributions. In Omey (2006) and Baltrunas, Van Gulck and Omey (2006, section 4) we studied the class of d.f. satisfying

$$
\frac{1-F^{* n}(\vec{x})}{1-F(\vec{x})} \rightarrow n
$$

as $x^{0}=\min \left(x_{1}, x_{2}, \ldots, x_{d}\right) \rightarrow \infty$. See also Daley, Omey and Vesilo (2007, Section 5). In dimension $d=1$, this is the usual subexponential class $S$. In Omey, Mallor and Santos (2006), we considered d.fs satisfying a relation of the form

$$
\lim _{t \rightarrow \infty} \frac{1-F^{* n}(t \vec{x}+\bar{a})}{1-F(t \vec{x})}=n, \quad \text { for each } \vec{a} \text { and each } \vec{x}>\overrightarrow{0}, \text { with } x^{0}<\infty \text {. }
$$

Using $R_{n}$ and $D_{n}$, we are able to study the rate of convergence in these definitions.

We are interested in two types of results. In the first place, we are interested in general inequalities and upper bounds. Secondly, we are interested in asymptotic equalities. In the next section, we briefly discuss the one-dimensional case, which has been studied, among others, by Omey (1994), Daley et al. (2007) and Baltrunas and Omey (1998).

\section{Results in the one-dimensional case}

In the one-dimensional case, we start from nonnegative random variables $X$ and $Y$ with d.f. $F$ and $G$, respectively. We are interested in the following quantities:

$$
\begin{array}{rlrl}
D(x) & =F(x) G(x)-F * G(x) ; & & D_{n}(x)=F^{n}(x)-F^{* n}(x) ; \\
R(x)=\overline{F * G}(x)-\bar{F}(x)-\bar{G}(x) ; & R_{n}(x)=\overline{F^{* n}}(x)-n \bar{F}(x) .
\end{array}
$$

To state some of the known results, we need the following classes of positive and measurable functions, cf. Omey (1994): (throughout, we assume limits as $x \rightarrow \infty$.) 
$f \in L: \quad$ for all $y$ we have $f(x+y) / f(x) \rightarrow 1$;

$f \in O D(m): \quad$ for all $y$ we have $|f(x+y)-f(x)|=O(1) m(x)$;

$f \in D(m, \alpha):$ for all $y$ we have $(f(x+y)-f(x)) / m(x) \rightarrow \alpha y$;

$f \in O R V: \quad$ for all $y>0$ we have $f(x y)=O(1) f(x)$;

$f \in R V(\alpha)$ : for all $y>0$ we have $f(x y) / f(x) \rightarrow y^{\alpha}$;

$f \in \Pi_{\alpha}(m): \quad$ for all $y>0$ we have $(f(x y)-f(x)) / m(x) \rightarrow \alpha \log (y)$.

Note that $f \in D(m, \alpha)$ with $\alpha \neq 0$ implies that $f(\log (x)) \in \Pi_{\alpha}(g(x))$, where $g(x)=m(\log (x)) \in R V(0)$. For a measurable function $f$, the upper and lower Matuszewska indices are defined as follows:

$$
\alpha(f)=\lim _{x \rightarrow \infty} \frac{\log \lim \sup _{t \rightarrow \infty} f(t x) / f(t)}{\log (x)},
$$

and

$$
\beta(f)=\lim _{x \rightarrow \infty} \frac{\log \liminf f(t x) / f(t)}{\log (x)} .
$$

It can be proved that $f \in O R V$ if and only if $\alpha(f)<\infty$ and $\beta(f)>-\infty$. Properties of these indices can be found in the books of Bingham et al. (1987) or Geluk and de Haan (1987).

We have the following representation theorem for the classes $O D(m)$ and $D(m, \alpha)$.

Theorem 2.1 (Omey 1994, 1995). (i)(Representation theorem for $O D(m)$ and $D(m, 0))$ Assume that $f \in O D(m)$, resp. $f \in D(m, 0)$ and suppose that $m(\log (x)) \in$ $O R V$. Then there exist constants $C$ and $x^{0}>0$ such that

$$
f(x)=C+\eta(x) m(x)+\int_{x^{0}}^{x} \phi(z) m(z) d z, \quad x \geqslant x^{0},
$$

where the measurable functions $\eta(x)$ and $\phi(x)$ are bounded, resp. $\eta(x)=o(1)$ and $\phi(x)=o(1)$.

(ii) (Representation theorem for $D(m, \alpha), \alpha \neq 0)$ Assume that $f \in D(m, \alpha)$ and suppose that $\alpha \neq 0$. Then there exist constants $C$ and $x^{0}>0$ such that

$$
f(x)=C+\eta(x) m(x)+\int_{x^{0}}^{x} \phi(z) m(z) d z, \quad x \geqslant x^{0},
$$

where $\eta$ and $\phi$ are measurable functions satisfying $\eta(x)=o(1)$ and $\phi(x) \rightarrow \alpha$.

The representation theorems can be used to obtain upper bounds.

Proposition 2.1 (Omey 1994, 1995). Suppose that $f \in O D(m)$ and assume that $m(x) \in O R V$. Then there exist constants $C$ and $x^{0}$ such that

$$
|f(x+y)-f(x)| \leqslant C(1+|y|) m(x), \quad x \geqslant x^{0}, \quad|y| \leqslant x / 2 .
$$

If $f \in D(m, 0)$, for each $\varepsilon>0$ we can find $x(\varepsilon)$ such that

$$
|f(x+y)-f(x)| \leqslant \varepsilon(1+|y|) m(x), \quad x \geqslant x(\varepsilon),|y| \leqslant x / 2 .
$$


If $F(x)$ is a d.f. such that $F \in O D(m)$, respectively $F \in D(m, 0)$, with $m \in$ $O R V$ and $\alpha(m)<-1$, then $\bar{F}(x)=O(1) x m(x)$, respectively $\bar{F}(x)=o(1) x m(x)$ (Omey, 1994, Prop. 3.1.1). In the proposition below, we use integrals of the form

$$
F_{1}(x)=\int_{0}^{x} y d F(y) .
$$

If $m \in O R V$, it is clear that $F \in O D(m)$ if and only if $F_{1} \in O D(M)$, where $M(x)=x m(x)$. If $m \in O R V$, with $\beta(m)>-2$, one can prove that $F_{1}(x) \rightarrow \infty$ and that $F_{1}(x)=O(1) x^{2} m(x)$.

Using these propositions, Omey (1994) proved the following results.

THEOREM 2.2. (a) Suppose that $F \in O D(m), G \in O D(n)$, with $m, n \in O R V$.

(i) We have $D(x)=O(1) m(x) G_{1}(x)+O(1) n(x) F_{1}(x)$.

(ii) If $\beta(n)>-2, \beta(m)>-2$, then $E(X)=E(Y)=\infty$ and $D(x)=$ $O(1) x^{2} n(x) m(x)$.

(iii) $\forall n \geqslant 2, D_{n}(x)=O(1) m(x) F_{1}(x)$. If $\beta(m)>-2$, then $D_{n}(x)=$ $O(1) x^{2} m^{2}(x)$.

(b) If $F \in D(m, 0), G \in D(n, 0)$, with $m, n \in O R V$, then the results of (a) hold with the $O(1)$-terms replaced by o(1)-terms.

(c) Suppose that $F \in O D(m)$ with $m \in O R V$.

(i) We have $R_{n}(x)=O(1) m(x) F_{1}(x)+O(1) \bar{F}^{2}(x)$.

(ii) If $\beta(m)>-2$, then $R_{n}(x)=O(1) x^{2} m^{2}(x)+O(1) \bar{F}^{2}(x)$.

(iii) If $\beta(m)>-2$ and $\alpha(m)<-1$, then $R_{n}(x)=O(1) x^{2} m^{2}(x)$.

Related results can be found in Baltrunas and Omey (1998) or Baltrunas et al. (2006).

In the next result, we reformulate some results related to asymptotic equalities.

Theorem 2.3 (Omey, 1994, 1995). Suppose that $F \in D(m, a), G \in D(n, b)$ with $m, n \in O R V \cap L$. Also, suppose that $E(X)+E(Y)<\infty$.

(i) We have $D(x)=a E(Y) m(x)+b E(X) n(x)+o(1) m(x)+o(1) n(x)$.

(ii) For all $n \geqslant 2$, we have $D_{n}(x)=2 a\left(\begin{array}{l}n \\ 2\end{array}\right) E(X) m(x)+o(1) m(x)$.

(iii) If $\alpha(m)<-1$, we have $R_{n}(x)=2 a\left(\begin{array}{c}n \\ 2\end{array}\right) E(X) m(x)+o(1) m(x)$.

\section{Results in the multidimensional case}

3.1. Inequalities. We denote by $F_{i}$ and $G_{i}(i=1, \ldots, d)$ the marginal distributions of $F$ and $G$ and let $D_{i}(x)=F_{i}(x) G_{i}(x)-F_{i} * G_{i}(x)$. The following lemma is easy to prove.

LEMmA 3.1. We have $0 \leqslant D(\vec{x}) \leqslant \sum_{i=1}^{d} D_{i}\left(x_{i}\right)$.

Proof. In the bivariate case, let $F(x, y)=P\left(X_{1} \leqslant x, X_{2} \leqslant y\right)$ and $G(x, y)=$ $P\left(Y_{1} \leqslant x, Y_{2} \leqslant y\right)$. We have $D(x, y)=I+I I$, where

$I=P\left(\max \left(X_{1}, Y_{1}\right) \leqslant x, \max \left(X_{2}, Y_{2}\right) \leqslant y\right)-P\left(X_{1}+Y_{1} \leqslant x, \max \left(X_{2}, Y_{2}\right) \leqslant y\right)$

and

$I I=P\left(X_{1}+Y_{1} \leqslant x, \max \left(X_{2}, Y_{2}\right) \leqslant y\right)-P\left(X_{1}+Y_{1} \leqslant x, X_{2}+Y_{2} \leqslant y\right)$. 
First consider $I$. Define the following events $A=\left\{X_{1}+Y_{1} \leqslant x\right\}$, $B=\left\{\max \left(X_{1}, Y_{1}\right) \leqslant x\right\}$ and $C=\left\{\max \left(X_{2}, Y_{2}\right) \leqslant y\right\}$. Since $A \subset B$, we have

$$
I=P(B \cap C)-P(A \cap C) \leqslant P(B)-P(A)=D_{1}(x) .
$$

In a similar way, we have $I I \leqslant D_{2}(y)$.

From Theorem 2.2, we have the following upper bounds for the marginals.

LEMma 3.2. Suppose that $m_{i}, n_{i} \in O R V$.

(i) If $F_{i} \in O D\left(m_{i}\right)$ and $G_{i} \in O D\left(n_{i}\right)$, then

$$
D_{i}(x)=O(1)\left(m_{i}(x) \int_{0}^{x} y d G_{i}(y)+n_{i}(x) \int_{0}^{x} y d F_{i}(y)\right) .
$$

(ii) If $F_{i} \in D\left(m_{i}, 0\right)$ and $G_{i} \in D\left(n_{i}, 0\right)$, then

$$
D_{i}(x)=o(1)\left(m_{i}(x) \int_{0}^{x} y d G_{i}(y)+n_{i}(x) \int_{0}^{x} y d F_{i}(y)\right) .
$$

Combining Lemmas 3.1 and 3.2, we obtain our first new result. We consider limits as $x^{0} \rightarrow \infty$.

TheOREM 3.1. Suppose that for each $i$ we have $m_{i}, n_{i} \in O R V$.

(i) Suppose that for each $i, F_{i} \in O D\left(m_{i}\right)$ and $G_{i} \in O D\left(n_{i}\right)$, then

$$
D(\vec{x})=O(1)\left(\sum_{i=1}^{d} m_{i}\left(x_{i}\right) \int_{0}^{x_{i}} y d G_{i}(y)+\sum_{i=1}^{d} n_{i}\left(x_{i}\right) \int_{0}^{x_{i}} y d F_{i}(y)\right) .
$$

(ii) Suppose that for each i, $F_{i} \in D\left(m_{i}, 0\right)$ and $G_{i} \in D\left(n_{i}, 0\right)$, then

$$
D(\vec{x})=o(1)\left(\sum_{i=1}^{d} m_{i}\left(x_{i}\right) \int_{0}^{x_{i}} y d G_{i}(y)+\sum_{i=1}^{d} n_{i}\left(x_{i}\right) \int_{0}^{x_{i}} y d F_{i}(y)\right) .
$$

To prove a result for $D_{n}(\vec{x})$, we need the following closure properties.

Lemma 3.3 (Omey 1994, Corollary 3.3.3). Suppose that $m_{i} \in O R V$.

(i) If $F_{i} \in O D\left(m_{i}\right)$ and $G_{i} \in O D\left(m_{i}\right)$, then $F_{i} * G_{i} \in O D\left(m_{i}\right)$ and $F_{i}^{* n} \in$ $O D\left(m_{i}\right)$.

(ii) If $F_{i} \in D\left(m_{i}, 0\right)$ and $G_{i} \in D\left(m_{i}, 0\right)$, then $F_{i} * G_{i} \in D\left(m_{i}, 0\right)$ and $F_{i}^{* n} \in$ $D\left(m_{i}, 0\right)$.

Now we can formulate a result for $D_{n}(\vec{x})$. Again, we consider statements as $x^{0} \rightarrow \infty$.

TheOREM 3.2. Suppose that for each $i$ we have $m_{i} \in O R V$.

(i) Suppose for each $i$ that $F_{i} \in O D\left(m_{i}\right)$; then for all $n \geqslant 2$,

$$
D_{n}(\vec{x})=O(1)\left(\sum_{i=1}^{d} m_{i}\left(x_{i}\right) \int_{0}^{x_{i}} y d F_{i}(y)\right) .
$$


(ii) Suppose for each $i$ that $F_{i} \in D\left(m_{i}, 0\right)$; then for all $n \geqslant 2$,

$$
D_{n}(\vec{x})=o(1)\left(\sum_{i=1}^{d} m_{i}\left(x_{i}\right) \int_{0}^{x_{i}} y d F_{i}(y)\right) .
$$

Proof. For $n=2$, this is the content of Theorem 3.1. Now let $G(\vec{x})=$ $F^{* n-1}(\vec{x})$ and $X_{i, j}(j=1, \cdots, n-1)$ be i.i.d. random variables with d.f. $F_{i}$. From Lemma 3.3 we have that $G_{i} \in O D\left(m_{i}\right)$. Also note that

$$
\begin{aligned}
\int_{0}^{x} y d G_{i}(y) & =E\left(I_{\left\{\sum_{j=1}^{n-1} X_{i, j} \leqslant x\right\}} \sum_{j=1}^{n-1} X_{i, j}\right)=(n-1) E\left(X_{i, 1} I_{\left\{\sum_{j=1}^{n-1} X_{i, j} \leqslant x\right\}}\right) \\
& \leqslant(n-1) E\left(X_{i, 1} I_{\left\{X_{i, 1} \leqslant x\right\}}\right)=(n-1) \int_{0}^{x} y d F_{i}(y) .
\end{aligned}
$$

Using Theorem 3.1 we obtain that

$$
G(\vec{x}) F(\vec{x})-F * G(\vec{x})=O(1)\left(\sum_{i=1}^{d} m_{i}\left(x_{i}\right) \int_{0}^{x_{i}} y d F_{i}(y)\right) .
$$

Now note that $D_{n}(\vec{x})=G(\vec{x}) F(\vec{x})-F * G(\vec{x})+F(\vec{x}) D_{n-1}(\vec{x})$. The proof now easily follows by induction on $n$.

If the means are finite, we obtain from Theorem $3.2(\mathrm{i})$ that

$$
D_{n}(\vec{x})=O(1)\left(\sum_{i=1}^{d} m_{i}\left(x_{i}\right)\right) \text {. }
$$

If $m_{i}(x) \in O R V$ with $-2<\beta\left(m_{i}\right)$, then the means are infinite and then (cf. Theorem 2.2) it follows that $D_{n, i}(x)=O(1) x_{i}^{2} m_{i}^{2}\left(x_{i}\right)$. In this case, Theorem 3.2 (i) gives

$$
D_{n}(\vec{x})=O(1)\left(\sum_{i=1}^{d} x_{i}^{2} m_{i}^{2}\left(x_{i}\right)\right) .
$$

In order to formulate a result about $R(\vec{x})$, recall that $R(\vec{x})=D(\vec{x})-\bar{F}(\vec{x}) \bar{G}(\vec{x})$. We have to deal with $\bar{F}(\vec{x}) \bar{G}(\vec{x})$. First note that $\bar{F}(\vec{x}) \leqslant \sum_{i=1}^{d} \bar{F}_{i}\left(x_{i}\right)$. Now suppose that $F_{i} \in O D\left(m_{i}\right), G_{i} \in O D\left(n_{i}\right)$, where $m_{i}, n_{i} \in O R V$ with $\alpha\left(m_{i}\right)<-1$ and $\alpha\left(n_{i}\right)<-1$. In this case, we have $\bar{F}_{i}\left(x_{i}\right)=O(1) x_{i} m_{i}\left(x_{i}\right)$ and $\vec{G}_{i}\left(x_{i}\right)=$ $O(1) x_{i} n_{i}\left(x_{i}\right)$, cf. (Omey 1994, Proposition 3.1.1(v)).

(a) In the finite means case, we find that $x_{i} \bar{F}_{i}\left(x_{i}\right) \rightarrow 0$, as $x_{i} \rightarrow \infty$, and it follows that $x^{0} \bar{F}(\vec{x}) \leqslant x^{0} \bar{F}\left(x^{0} \overrightarrow{1}\right) \leqslant x^{0} \sum_{i=1}^{d} \bar{F}_{i}\left(x^{0}\right) \rightarrow 0$, as $x^{0} \rightarrow \infty$. Hence, as $x^{0} \rightarrow \infty$, we obtain that

$$
\begin{aligned}
\bar{F}(\vec{x}) \bar{G}(\vec{x}) & \leqslant \bar{F}\left(x^{0} \overrightarrow{1}\right) \bar{G}\left(x^{0} \overrightarrow{1}\right) \leqslant \bar{F}\left(x^{0} \overrightarrow{1}\right) \sum_{i=1}^{d} x^{0} n_{i}\left(x^{0}\right) \\
& =x^{0} \bar{F}\left(x^{0} \overrightarrow{1}\right) \sum_{i=1}^{d} n_{i}\left(x^{0}\right)=o(1) \sum_{i=1}^{d} n_{i}\left(x^{0}\right) .
\end{aligned}
$$


In a similar way, we have $\bar{F}(\vec{x}) \bar{G}(\vec{x})=o(1) \sum_{i=1}^{d} m_{i}\left(x^{0}\right)$.

(b) If $-2<\beta\left(m_{i}\right)$ and $\alpha\left(m_{i}\right)<-1$, then $E\left(X_{i}\right)=\infty$, and we have

$$
\int_{0}^{x_{i}} y d F_{i}(y)=O(1) x_{i}^{2} m_{i}\left(x_{i}\right) .
$$

In this case, it follows that

$$
\begin{aligned}
\bar{F}(\vec{x}) \bar{G}(\vec{x}) & =O(1) \sum_{i=1}^{d} x^{0} m_{i}\left(x^{0}\right) \sum_{i=1}^{d} x^{0} n_{i}\left(x^{0}\right), \\
D(\vec{x}) & =O(1)\left(\sum_{i=1}^{d} x_{i}^{2} m_{i}\left(x_{i}\right) n_{i}\left(x_{i}\right)\right) .
\end{aligned}
$$

We conclude

Corollary 3.1. Suppose $m_{i}, n_{i} \in O R V$ with $\alpha\left(m_{i}\right)<-1, \alpha\left(n_{i}\right)<-1$.

(i) If $F_{i} \in O D\left(m_{i}\right), G_{i} \in O D\left(n_{i}\right)$ with finite means, then

$$
R(\vec{x})=O(1)\left(\sum_{i=1}^{d} m_{i}\left(x_{i}\right)+\sum_{i=1}^{d} n_{i}\left(x_{i}\right)\right)+o(1)\left(\sum_{i=1}^{d} m_{i}\left(x^{0}\right)+\sum_{i=1}^{d} n_{i}\left(x^{0}\right)\right) .
$$

(ii) If also $-2<\beta\left(m_{i}\right),-2<\beta\left(n_{i}\right)$, we have

$R(\vec{x})=O(1)\left(\sum_{i=1}^{d} x_{i}^{2} m_{i}\left(x_{i}\right) n_{i}\left(x_{i}\right)\right)+O(1)\left(\sum_{i=1}^{d} x^{0} m_{i}\left(x^{0}\right)\right)\left(\sum_{i=1}^{d} x^{0} n_{i}\left(x^{0}\right)\right)$.

(iii) Similar results hold if we start from $F_{i} \in D\left(m_{i}, 0\right)$ and $G_{i} \in D\left(n_{i}, 0\right)$.

For $R_{n}(\vec{x})$, the analysis is similar. Now we obtain the following result.

Corollary 3.2. Suppose that $m_{i} \in O R V$ with $\alpha\left(m_{i}\right)<-1$.

(i) If $F_{i} \in O D\left(m_{i}\right)$ with finite means, then for all $n \geqslant 2$,

$$
R_{n}(\vec{x})=O(1) \sum_{i=1}^{d} m_{i}\left(x_{i}\right)+o(1) \sum_{i=1}^{d} m_{i}\left(x^{0}\right) .
$$

(ii) If also $-2<\alpha\left(m_{i}\right)$, we have

$$
R_{n}(x)=O(1) \sum_{i=1}^{d} x_{i}^{2} m_{i}^{2}\left(x_{i}\right)+O(1)\left(\sum_{i=1}^{d} x^{0} m_{i}\left(x^{0}\right)\right)^{2} .
$$

(iii) Similar results hold if we start from $F_{i} \in D\left(m_{i}, 0\right)$.

3.2. Asymptotic equalities. In this section, we are seeking an asymptotic equality in the place of an upper bound. Our starting point is the following useful inequality (cf. Lemma 3.4). We suppose that $\vec{X}$ and $\vec{Y}$ are independent nonnegative random vectors with distribution function $F(\vec{x})$ and $G(\vec{x})$ respectively. We give an estimate for the difference $D(\vec{x})=F(\vec{x}) G(\vec{x})-F * G(\vec{x})$. 
LEMMA 3.4. We have $D(\vec{x})=A(\vec{x})+B(\vec{x})+C(\vec{x})$, where

$$
\begin{aligned}
A(\vec{x}) & =\int_{\overrightarrow{0}}^{\vec{x} / 2}(F(\vec{x})-F(\vec{x}-\vec{y})) d G(\vec{y}), \\
B(\vec{x}) & =\int_{\overrightarrow{0}}^{\vec{x} / 2}(G(\vec{x})-G(\vec{x}-\vec{y})) d F(\vec{y}), \\
|C(\vec{x})| & \leqslant 2(F(\vec{x})-F(\vec{x} / 2))(G(\vec{x})-G(\vec{x} / 2)) .
\end{aligned}
$$

Proof. We rewrite $F * G(\vec{x})$ as follows. We have

$$
\begin{aligned}
F * G(\vec{x})= & P(\vec{X}+\vec{Y} \leqslant \vec{x}, \vec{X} \leqslant \vec{x} / 2, \vec{Y} \leqslant \vec{x} / 2) \\
& +P\left(\vec{X}+\vec{Y} \leqslant \vec{x},\{\vec{X} \leqslant \vec{x} / 2\}^{c}, \vec{Y} \leqslant \vec{x} / 2\right) \\
& +P\left(\vec{X}+\vec{Y} \leqslant \vec{x}, \vec{X} \leqslant \vec{x} / 2,\{\vec{Y} \leqslant \vec{x} / 2\}^{c}\right) \\
& +P\left(\vec{X}+\vec{Y} \leqslant \vec{x},\{\vec{X} \leqslant \vec{x} / 2\}^{c},\{\vec{Y} \leqslant \vec{x} / 2\}^{c}\right) \\
= & I+I I+I I I+I V .
\end{aligned}
$$

For $I$, it is easy to see that $I=F(\vec{x} / 2) G(\vec{x} / 2)$. For $I I$, we have

$$
\begin{aligned}
I I & =\int_{\overrightarrow{0}}^{\vec{x} / 2} P\left(\vec{X} \leqslant \vec{x}-\vec{y},\{\vec{X} \leqslant \vec{x} / 2\}^{c}\right) d G(\vec{y}) \\
& =\int_{\overrightarrow{0}}^{\vec{x} / 2}(F(\vec{x}-\vec{y})-F(\vec{x} / 2)) d G(\vec{y}) \\
& =-A(\vec{x})+\int_{\overrightarrow{0}}^{\vec{x} / 2}(F(\vec{x})-F(\vec{x} / 2)) d G(\vec{y}) \\
& =-A(\vec{x})+(F(\vec{x})-F(\vec{x} / 2)) G(\vec{x} / 2) .
\end{aligned}
$$

In a similar way, we obtain that $I I I=-B(\vec{x})+(G(\vec{x})-G(\vec{x} / 2)) F(\vec{x} / 2)$. Hence, we obtain that

$$
\begin{aligned}
D(\vec{x}) & =F(\vec{x}) G(\vec{x})-F(\vec{x} / 2) G(\vec{x} / 2)+A(\vec{x})+B(\vec{x}) \\
& -(F(\vec{x})-F(\vec{x} / 2)) G(\vec{x} / 2)-(G(\vec{x})-G(\vec{x} / 2)) F(\vec{x} / 2)-I V \\
& =(F(\vec{x})-F(\vec{x} / 2))(G(\vec{x})-G(\vec{x} / 2))+A(\vec{x})+B(\vec{x})-I V .
\end{aligned}
$$

Now note that in $I V$ we have $\{\vec{X}+\vec{Y} \leqslant \vec{x}\} \subset\{\vec{X} \leqslant \vec{x}\} \cap\{\vec{Y} \leqslant \vec{x}\}$ and we find that

$$
\begin{aligned}
I V & \leqslant P\left(\vec{X} \leqslant \vec{x}, \vec{Y} \leqslant \vec{x} / 2,\{\vec{X} \leqslant \vec{x} / 2\}^{c},\{\vec{Y} \leqslant \vec{x} / 2\}^{c}\right) \\
& \leqslant P\left(\vec{X} \leqslant \vec{x},\{\vec{X} \leqslant \vec{x} / 2\}^{c}\right) P\left(\vec{Y} \leqslant \vec{x} / 2,\{\vec{Y} \leqslant \vec{x} / 2\}^{c}\right) \\
& =(F(\vec{x})-F(\vec{x} / 2))(G(\vec{x})-G(\vec{x} / 2)) .
\end{aligned}
$$

This proves the result. 
3.2.1. The class $D_{d}(m, \lambda)$. Now we introduce a multivariate analogue of the class $D(m, \alpha)$.

Definition 1. We say that the d.f. $F$ is in the class $\mathfrak{D}_{d}(m, \lambda)$ if we have

$$
\frac{F(t \vec{x})-F(t \vec{x}-\vec{a})}{m(t)} \rightarrow \lambda(\vec{x}, \vec{a}), \quad t \rightarrow \infty,
$$

for all $\vec{x}>\overrightarrow{0}$, with $x^{0}<\infty$ and $\vec{a} \in \mathbb{R}^{d}$. The function $\lambda(\vec{x}, \vec{a})$ is called the limit function.

Note that in the definition, we assume that the defining property holds for each of the marginals of $F$. For the $i$-th marginal we have

$$
\frac{F_{i}(t x)-F_{i}(t x-a)}{m(t)} \rightarrow \lambda_{i}(x, a), \text { for each } x>0, \text { and each } a \in \mathbb{R} \text {. }
$$

By taking $x=1$, we obtain that

$$
\frac{F_{i}(t)-F_{i}(t-a)}{m(t)} \rightarrow \lambda_{i}(1, a), \text { for each } x>0, \text { and each } a \in \mathbb{R} .
$$

If $m \in L$,we obtain that

$$
\begin{aligned}
\frac{F_{i}(t)-F_{i}(t-a-b)}{m(t)} & =\frac{F_{i}(t)-F_{i}(t-a)}{m(t)}+\frac{F_{i}(t-a)-F_{i}(t-a-b)}{m(t-a)} \frac{m(t-a)}{m(t)} \\
& \rightarrow \lambda_{i}(1, a)+\lambda_{i}(1, b) .
\end{aligned}
$$

It follows that $\lambda_{i}(1, a+b)=\lambda_{i}(1, a)+\lambda_{i}(1, b)$, and then also that $\lambda_{i}(1, a)=\alpha_{i} a$ for some real constant $\alpha_{i}$.

Now observe that

$$
\frac{F_{i}(t x)-F_{i}(t x-a)}{m(t)}=\frac{F_{i}(t x)-F_{i}(t x-a)}{m(t x)} \frac{m(t x)}{m(t)} .
$$

After taking limits, we obtain that

$$
\lambda_{i}(x, a)=\alpha_{i} a \lim _{t \rightarrow \infty} \frac{m(t x)}{m(t)} .
$$

If $\alpha_{i} \neq 0$ and $a \neq 0$, we obtain that

$$
\lim _{t \rightarrow \infty} \frac{m(t x)}{m(t)}=\frac{\lambda_{i}(x, a)}{\alpha_{i} a} .
$$

In this case, it follows under minimal conditions, that $m \in R V(\delta)$ for some real number $\delta$, and as a consequence, that $\lambda_{i}(x, a)=\alpha_{i} x^{\delta} a$.

In what follows, we will assume that in the definition of $\mathfrak{D}_{d}(\lambda, m), m$ is a regularly varying function.

Definition 2. We say that the d.f. $F$ is in the class $D_{d}(m, \lambda)$ if we have $m \in R V(\delta)$ and if

$$
\frac{F(t \vec{x})-F(t \vec{x}-\vec{a})}{m(t)} \rightarrow \lambda(\vec{x}, \vec{a}), \quad t \rightarrow \infty,
$$


for all $\vec{x}>\overrightarrow{0}$, with $x^{0}<\infty$ and $\vec{a} \in \mathbb{R}^{d}$. The function $\lambda(\vec{x}, \vec{a})$ is called the limit function. In this case, the marginals $F_{i} \in D\left(m, \lambda_{i}\right)$, where $\lambda_{i}(x, a)=\alpha_{i} a x^{\delta}$.

In the next section, it will be convenient to assume that $F \in D_{d}(m, \lambda)$, and that the defining property holds locally uniformly in $\vec{x}$.

Definition 3. We say that the d.f. $F$ is in the class $D L_{d}(m, \lambda)$ if we have $m \in R V(\delta)$ and if

$$
\frac{F(t \vec{x})-F(t \vec{x}-\vec{a})}{m(t)} \rightarrow \lambda(\vec{x}, \vec{a}), \quad t \rightarrow \infty,
$$

for all $\vec{x}>\overrightarrow{0}$, with $x^{0}<\infty$ and $\vec{a} \in \mathbb{R}^{d}$, holds locally uniformly in $\vec{x}$.

In this case, we can show that the limit function is an additive function in $\vec{a}$. To show this, note that we have

$$
\frac{F(t \vec{x})-F(t \vec{x}-\vec{a}-\vec{b})}{m(t)} \rightarrow \lambda(\vec{x}, \vec{a}+\vec{b}) .
$$

On the other hand, we also have that

$$
\begin{aligned}
\frac{F(t \vec{x})-F(t \vec{x}-\vec{a}-\vec{b})}{m(t)} & =\frac{F(t \vec{x})-F(t \vec{x}-\vec{a})}{m(t)}+\frac{F(t \vec{x}-\vec{a})-F(t \vec{x}-\vec{a}-\vec{b})}{m(t)} \\
& =\frac{F(t \vec{x})-F(t \vec{x}-\vec{a})}{m(t)}+\frac{F(t(\vec{x}-\vec{a} / t))-F(t(\vec{x}-\vec{a} / t)-\vec{b})}{m(t)} \\
& \rightarrow \lambda(\vec{x}, \vec{a})+\lambda(\vec{x}, \vec{b})
\end{aligned}
$$

and it follows that $\lambda(\vec{x}, \vec{a}+\vec{b})=\lambda(\vec{x}, \vec{a})+\lambda(\vec{x}, \vec{b})$.

3.2.2. Main result. This is the main result of this section. Recall that $\vec{X}$ has d.f. $F$ and $\vec{Y}$ has d.f. $G$.

Theorem 3.3. Suppose that $F \in D_{d}(m, \lambda), G \in D_{d}(n, \theta)$ have finite means and that $m, n \in R V$. Then

$$
\begin{array}{r}
D(t \vec{x}-\vec{a})=[E \lambda(\vec{x}, \vec{a}+\vec{Y})-\lambda(\vec{x}, \vec{a})] m(t)+[E \theta(\vec{x}, \vec{a}+\vec{X})-\theta(\vec{x}, \vec{a})] n(t) \\
+o(1) n(t)+o(1) m(t) .
\end{array}
$$

Proof. We analyze the three terms in Lemma 3.4 First, take $A(\vec{x})$ and replace $\vec{x}$ by $t \vec{x}-\vec{a}$. We have

$$
\begin{aligned}
A(t \vec{x}-\vec{a})= & \int_{\overrightarrow{0}}^{(t \vec{x}-\vec{a}) / 2}(F(t \vec{x}-\vec{a})-F(t \vec{x}-\vec{a}-\vec{y})) d G(\vec{y}) \\
= & (F(t \vec{x}-\vec{a})-F(t \vec{x})) G((t \vec{x}-\vec{a}) / 2) \\
& +\int_{\overrightarrow{0}}^{(t \vec{x}-\vec{a}) / 2}(F(t \vec{x})-F(t \vec{x}-\vec{a}-\vec{y})) d G(\vec{y}) \\
= & I+I I .
\end{aligned}
$$


We first analyze $I I$. Clearly, we have

$$
\frac{F(t \vec{x})-F(t \vec{x}-\vec{a}-\vec{y})}{m(t)} \rightarrow \lambda(\vec{x}, \vec{a}+\vec{y}) .
$$

We want to apply Lebesgue's theorem on dominated convergence. Clearly, we have

$$
F(t \vec{x})-F(t \vec{x}-\vec{a}-\vec{y}) \leqslant \sum_{i=1}^{d}\left(F_{i}\left(t x_{i}\right)-F_{i}\left(t x_{i}-a_{i}-y_{i}\right)\right)
$$

and $0 \leqslant y_{i} \leqslant\left(t x_{i}-a_{i}\right) / 2$. Using Proposition 2.1, we get that

$$
F_{i}\left(t x_{i}\right)-F_{i}\left(t x_{i}-a_{i}-y_{i}\right) \leqslant C_{i}\left(1+\left|a_{i}+y_{i}\right|\right) m\left(t x_{i}\right), \quad t x_{i} \geqslant x^{*} .
$$

Using $m \in R V$, and since $\vec{x}$ is fixed, we find that for $\vec{y} \leqslant(t \vec{x}-\vec{a}) / 2$ and $t$ sufficiently large, we have

$$
|F(t \vec{x})-F(t \vec{x}-\vec{a}-\vec{y})| \leqslant \sum_{i=1}^{d} C_{i}\left(1+\left|a_{i}+y_{i}\right|\right) m(t) .
$$

Since, by assumption, the means $E\left(Y_{i}\right)$ are finite, we can apply Lebesgue's theorem on dominated convergence, and we conclude that

$$
\frac{1}{m(t)} A(t \vec{x}-\vec{a}) \rightarrow-\lambda(\vec{x}, \vec{a})+E \lambda(\vec{x}, \vec{a}+\vec{Y}) .
$$

In a similar way, we obtain that

$$
\frac{1}{n(t)} B(t \vec{x}-\vec{a}) \rightarrow-\theta(\vec{x}, \vec{a})+E \theta(\vec{x}, \vec{a}+\vec{X}) .
$$

For $C(t \vec{x}-\vec{a})$, we proceed as follows. First, we have that, cf. Proposition 2.1

$$
\begin{aligned}
F(t \vec{x}-\vec{a})-F((t \vec{x}-\vec{a}) / 2) & \leqslant \sum_{i=1}^{d}\left(F_{i}\left(t x_{i}-a_{i}\right)-F_{i}\left(\left(t x_{i}-a_{i}\right) / 2\right)\right) \\
& =O(1) \sum_{i=1}^{d} t x_{i} m\left(t x_{i}\right)=O(1) t m(t) .
\end{aligned}
$$

Also, we have that

$$
G(t \vec{x}-\vec{a})-G((t \vec{x}-\vec{a}) / 2) \leqslant \sum_{i=1}^{d}\left(G_{i}\left(t x_{i}-a_{i}\right)-G_{i}\left(\left(t x_{i}-a_{i}\right) / 2\right)\right)=o(1) t^{-1},
$$

since the means are assumed to be finite. It follows that $C(t \vec{x}-\vec{a})=o(1) n(t)$. By changing the role of $F$ and $G$, we can also deduce that $C(t \vec{x}-\vec{a})=o(1) m(t)$. Now, we can combine the estimates. This proves the result.

In the special case that $m=n$, we find that

$$
\frac{D(t \vec{x}-\vec{a})}{m(t)} \rightarrow E[\lambda(\vec{x}, \vec{a}+\vec{Y})]-\lambda(\vec{x}, \vec{a})+E[\theta(\vec{x}, \vec{a}+\vec{X})]-\theta(\vec{x}, \vec{a}) .
$$

Now note that $F * G=D+F G$. If $m=n$, it follows from Theorem 3.3 that 


$$
\begin{aligned}
& \frac{F * G(t \vec{x}-\vec{a})-F * G(t \vec{x})}{m(t)}=\frac{D(t \vec{x}-\vec{a})-D(t \vec{x})}{m(t)} \\
& \quad+\frac{(F(t \vec{x}-\vec{a})-F(t \vec{x})) G(t \vec{x}-\vec{a})}{m(t)}+\frac{F(t \vec{x})(G(t \vec{x}-\vec{a})-G(t \vec{x}))}{m(t)} \\
& \rightarrow E[\lambda(\vec{x}, \vec{a}+\vec{Y})-\lambda(\vec{x}, \vec{Y})]+E[\theta(\vec{x}, \vec{a}+\vec{X})-\theta(\vec{x}, \vec{X})]
\end{aligned}
$$

As a consequence, we have $F * G \in D(m, \xi)$ for some limit function $\xi$.

Taking $F=G$, we get that

$$
\frac{F^{* 2}(t \vec{x}-\vec{a})-F^{* 2}(t \vec{x})}{m(t)}=2 E[\lambda(\vec{x}, \vec{a}+\vec{X})-\lambda(\vec{x}, \vec{X})] .
$$

For convenience, we set

$$
\lambda_{1}(\vec{x}, \vec{a})=\lambda(\vec{x}, \vec{a}), \quad \lambda_{2}(\vec{x}, \vec{a})=2 E[\lambda(\vec{x}, \vec{a}+\vec{X})-\lambda(\vec{x}, \vec{X})] .
$$

Now let $\vec{X}_{1}, \vec{X}_{2}, \ldots, \vec{X}_{n}$ denote i.i.d. copies of $\vec{X}$ and for $i \geqslant 1$, let $\vec{S}_{i}=\vec{X}_{1}+\cdots+\vec{X}_{i}$. Taking $G=F^{* 2}$ in expression (1), we obtain that

$$
\begin{aligned}
\frac{F^{* 3}(t \vec{x}-\vec{a})-F^{* 3}(t \vec{x})}{m(t)=} & E\left[\lambda_{2}\left(\vec{x}, \vec{a}+\vec{S}_{2}\right)-\lambda_{2}\left(\vec{x}, \vec{S}_{2}\right)\right] \\
& +E\left[\lambda_{1}(\vec{x}, \vec{a}+\vec{X})-\lambda_{1}(\vec{x}, \vec{X})\right] \\
\equiv & \lambda_{3}(\vec{x}, \vec{a}) .
\end{aligned}
$$

Proceeding in a similar way, we take $G=F^{* n-1}$ in (1) to obtain that

$$
\begin{aligned}
\frac{F^{* n}(t \vec{x}-\vec{a})-F^{* n}(t \vec{x})}{m(t)}= & E\left[\lambda_{n-1}\left(\vec{x}, \vec{a}+\vec{S}_{n-1}\right)-\lambda_{n-1}\left(\vec{x}, \vec{S}_{n-1}\right)\right] \\
& +E\left[\lambda_{1}(\vec{x}, \vec{a}+\vec{X})-\lambda_{1}(\vec{x}, \vec{X})\right] \\
\equiv & \lambda_{n}(\vec{x}, \vec{a}) .
\end{aligned}
$$

We have proved the following result.

Corollary 3.3. Suppose that $F \in D_{d}(m, \lambda)$ has a finite mean and that $m \in R V$. Then, for each $n \geqslant 2$, we have $F^{* n} \in D_{d}\left(m, \lambda_{n}\right)$, where $\lambda_{n}$ is defined recursively above.

If the defining property of $F \in D_{d}(m, \lambda)$ holds locally uniformly in $\vec{x}$ we have (cf. previous section) that $\lambda(\vec{x}, \vec{a}+\vec{b})=\lambda(\vec{x}, \vec{a})+\lambda(\vec{x}, \vec{b})$ and we can simplify the expressions for $\lambda_{n}$. We clearly have

$$
\begin{aligned}
\lambda_{2}(\vec{x}, \vec{a})= & 2 E[\lambda(\vec{x}, \vec{a}+\vec{X})-\lambda(\vec{x}, \vec{X})]=2 \lambda(\vec{x}, \vec{a}), \\
\lambda_{3}(\vec{x}, \vec{a})= & E\left[\lambda_{2}\left(\vec{x}, \vec{a}+\vec{X}_{1}+\vec{X}_{2}\right)-\lambda_{2}\left(\vec{x}, \vec{X}_{1}+\vec{X}_{2}\right)\right] \\
& +E\left[\lambda_{1}(\vec{x}, \vec{a}+\vec{X})-\lambda_{1}(\vec{x}, \vec{X})\right] \\
= & 2 E\left[\lambda\left(\vec{x}, \vec{a}+\vec{X}_{1}+\vec{X}_{2}\right)-\lambda\left(\vec{x}, \vec{X}_{1}+\vec{X}_{2}\right)\right] \\
& +\lambda(\vec{x}, \vec{a})=3 \lambda(\vec{x}, \vec{a}) .
\end{aligned}
$$

The final result is that $\lambda_{n}(\vec{x}, \vec{a})=n \lambda(\vec{x}, \vec{a})$. 
3.2.3. A result for $D_{n}$. Now, we consider $D_{n}(\vec{x})$. Clearly, we have

$$
\begin{aligned}
D_{2}(\vec{x}) & =F^{2}(\vec{x})-F^{* 2}(\vec{x}), \\
D_{n+1}(\vec{x}) & =F^{n+1}(\vec{x})-F^{* n+1}(\vec{x})=F(\vec{x}) D_{n}(\vec{x})+D(\vec{x}),
\end{aligned}
$$

where $D(\vec{x})=F(\vec{x}) G(\vec{x})-F * G(\vec{x})$ and $G(\vec{x})=F^{* n}(x)$.

As before, let let $\vec{X}_{1}, \vec{X}_{2}, \ldots, \vec{X}_{n}$ denote i.i.d. copies of $\vec{X}$, and let $\vec{S}_{j}=\vec{X}_{1}+$ $\vec{X}_{2}+\cdots+\vec{X}_{j}$.

From Theorem 3.3 and Corollary 3.3 we have that $G(\vec{x}) \in D_{d}\left(m, \lambda_{n}\right)$ and

$$
\frac{1}{m(t)} D(t \vec{x}-\vec{a}) \rightarrow E\left[\lambda\left(\vec{x}, \vec{a}+\vec{S}_{n}\right)\right]-\lambda(\vec{x}, \vec{a})+E\left[\lambda_{n}(\vec{x}, \vec{a}+\vec{X})\right]-\lambda_{n}(\vec{x}, \vec{a}) .
$$

From Theorem 3.3, we also have that

$$
\frac{1}{m(t)} D_{2}(t \vec{x}-\vec{a}) \rightarrow 2 E(\lambda(\vec{x}, \vec{a}+\vec{X})-\lambda(\vec{x}, \vec{a}))=\Psi_{2}(\vec{x}, \vec{a}) .
$$

It follows that $\frac{1}{m(t)} D_{n+1}(t \vec{x}-\vec{a}) \rightarrow \Psi_{n+1}(\vec{x}, \vec{a})$, where

$$
\Psi_{n+1}(\vec{x}, \vec{a})=\Psi_{n}(\vec{x}, \vec{a})+E\left[\lambda\left(\vec{x}, \vec{a}+\vec{S}_{n}\right)\right]-\lambda(\vec{x}, \vec{a})+E\left[\lambda_{n}(\vec{x}, \vec{a}+\vec{X})\right]-\lambda_{n}(\vec{x}, \vec{a}) .
$$

In the case where $\lambda(\vec{x}, \vec{a}+\vec{b})=\lambda(\vec{x}, \vec{a})+\lambda(\vec{x}, \vec{b})$, we have

$$
\begin{aligned}
\Psi_{2}(\vec{x}, \vec{a}) & =2 E \lambda(\vec{x}, \vec{X}), \\
\Psi_{n+1}(\vec{x}, \vec{a}) & =\Psi_{n}(\vec{x}, \vec{a})+E \lambda\left(\vec{x}, \vec{S}_{n}\right)+E \lambda_{n}(\vec{x}, \vec{X})=\Psi_{n}(\vec{x}, \vec{a})+2 n E \lambda(\vec{x}, \vec{X}) .
\end{aligned}
$$

It easily follows by induction that $\Psi_{n}(\vec{x}, \vec{a})=2\left(\begin{array}{c}n \\ 2\end{array}\right) E \lambda(\vec{x}, \vec{a})$. Summarizing, we have proved the following result

Corollary 3.4. Suppose that $F \in D_{d}(m, \lambda)$ has a finite mean and that $m \in$ $R V$. Then, for each $n \geqslant 2$, we have $\frac{1}{m(t)} D_{n}(t \vec{x}-\vec{a}) \rightarrow \Psi_{n}(\vec{x}, \vec{a})$, where $\Psi_{n}$ is defined recursively above. Moreover, if $F \in D L_{d}(m, \lambda)$, we have $\Psi_{n}(\vec{x}, \vec{a})=2\left(\begin{array}{c}n \\ 2\end{array}\right) E \lambda(\vec{x}, \vec{a})$.

In the case of $R_{n}$, we have the following result.

Corollary 3.5. Suppose that $F \in D_{d}(m, \lambda)$ has a finite mean and that $m \in$ $R V$. Also, assume that

$$
\frac{(1-F(t \vec{x}-\vec{a}))^{2}}{m(t)} \rightarrow 0 \text {, for all } \vec{x}>\overrightarrow{0} \text { with } x^{0}<\infty \text { and } \vec{a} \in \mathbb{R}^{d} \text {. }
$$

Then, for each $n \geqslant 2$, we have $\frac{1}{m(t)} R_{n}(t \vec{x}-\vec{a}) \rightarrow \Psi_{n}(\vec{x}, \vec{a})$, where $\Psi_{n}$ is defined recursively above.

3.2.4. Examples. ExAmple 1. For simplicity, we consider d.fs in $\mathbb{R}^{2}$. We say that a positive and measurable function $f$ is regularly varying in the sense of Yakymiv (1982) if there exists function $h \in R V$ such that $\frac{f(t x, t y)}{h(t)} \rightarrow \lambda(x, y)$ locally uniformly in $(x, y)>(0,0)$. Notation: $f \in R V_{Y}(h, \lambda)$. We say a function of several variables is monotone if it is increasing or decreasing in each variable. If $f$ is monotone, then pointwise convergence automatically implies convergence locally uniformly. 
In our first example, we assume that the d.f. $F$ has first partial derivatives $f_{1}$ and $f_{2}$ that are both regularly varying in the sense of Yakymiv, with the same auxiliary function $m \in R V$. Now we write

$$
\begin{aligned}
F(t x, t y) & -F(t x-a, t y-b) \\
& =\int_{t x-a}^{t x} f_{1}(u, t y) d u+\int_{t y-b}^{t y} f_{2}(t x-a, w) d w \\
& =\int_{-a}^{0} f_{1}(t x+u, t y) d u+\int_{-b}^{0} f_{2}(t x-a, t y+w) d w .
\end{aligned}
$$

By our assumption, we have $f_{i}(t x, t y) / m(t) \rightarrow \lambda_{i}(x, y)$, locally uniformly in $(x, y)>(0,0)$. Considering the term with $f_{1}$ and using

$$
\frac{f_{1}(t x+u, t y)}{m(t)}=\frac{f_{1}(t(x+u / t), t y)}{m(t)} \rightarrow \lambda_{1}(x, y),
$$

and, similarly, for the term containing $f_{2}$, we find that

$$
\frac{F(t x, t y)-F(t x-a, t y-b)}{m(t)} \rightarrow a \lambda_{1}(x, y)+b \lambda_{2}(x, y) \text {. }
$$

If also the marginals are in some class $D\left(m, \alpha_{i}\right)$, we obtain that $F \in D_{2}(m, \lambda)$, where $\lambda(x, y)=a \lambda_{1}(x, y)+b \lambda_{2}(x, y)$.

ExAmple 2. Assume $F(x, y)=F_{1}(x) F_{2}(y)\left(1+\theta \bar{F}_{1}(x) \bar{F}_{2}(y)\right)$. We have

$$
\begin{aligned}
F(x+ & a, y+b)-F(x, y) \\
= & F_{1}(x+a) F_{2}(y+b)\left(1+\theta \bar{F}_{1}(x+a) \bar{F}_{2}(y+b)\right) \\
& -F_{1}(x) F_{2}(y)\left(1+\theta \bar{F}_{1}(x) \bar{F}_{2}(y)\right) \\
= & \left(F_{1}(x+a)-F_{1}(x)\right) F_{2}(y+b)\left(1+\theta \bar{F}_{1}(x+a) \bar{F}_{2}(y+b)\right) \\
& +F_{1}(x)\left(F_{2}(y+b)-F_{2}(y)\right)\left(1+\theta \bar{F}_{1}(x+a) \bar{F}_{2}(y+b)\right) \\
& +\theta F_{1}(x) F_{2}(y)\left(\bar{F}_{1}(x+a) \bar{F}_{2}(y+b)-\bar{F}_{1}(x) \bar{F}_{2}(y)\right) \\
= & (1+o(1))\left(F_{1}(x+a)-F_{1}(x)+F_{2}(y+b)-F_{2}(y)\right) \\
& +\theta(1+o(1))\left(\left(\bar{F}_{1}(x+a)-\bar{F}_{1}(x)\right) \bar{F}_{2}(y+b)+\bar{F}_{1}(x)\left(\bar{F}_{2}(y+b)-\bar{F}_{2}(y)\right)\right) \\
= & (1+o(1))\left(F_{1}(x+a)-F_{1}(x)+F_{2}(y+b)-F_{2}(y)\right) \\
& +\theta(1+o(1))\left(\left(F_{1}(x+a)-F_{1}(x)\right) \bar{F}_{2}(y+b)+\bar{F}_{1}(x)\left(F_{2}(y+b)-F_{2}(y)\right)\right) .
\end{aligned}
$$

If $F_{1} \in D(m, \alpha)$ and $F_{2} \in D(m, \beta)$, where $m \in R V(\lambda)$, we obtain that

$$
\begin{gathered}
F(t x+a, t y+b)-F(t x, t y) \\
=(1+o(1))\left(F_{1}(t x+a)-F_{1}(t x)+F_{2}(t y+b)-F_{2}(t y)\right) \\
+\theta(1+o(1))\left(\left(F_{1}(t x+a)-F_{1}(t x)\right) \bar{F}_{2}(t y+b)\right. \\
\left.+\bar{F}_{1}(t x)\left(F_{2}(t y+b)-F_{2}(t y)\right)\right)
\end{gathered}
$$


and then

$$
\frac{F(t x+a, t y+b)-F(t x, t y)}{m(t)} \rightarrow \alpha a x^{\lambda}+\beta b y^{\lambda}, \quad \min (x, y)<\infty .
$$

EXAmPle 3. Now suppose that

$$
F(x, y)=\frac{F_{1}(x) F_{2}(y)}{1+\theta \bar{F}_{1}(x) \bar{F}_{2}(y)} .
$$

We obtain a similar result to Example 2.

ExAmple 4. Suppose the bivariate distribution function, $F$, of $(X, Y)$ is given by the copula function $\Psi$ defined by $F(x, y)=\Psi\left(F_{1}(x), F_{2}(y)\right)$, where $F_{1}$ and $F_{2}$ are the marginal distribution functions of $X$ and $Y$, respectively.

Suppose $F_{1} \in D_{d}\left(m, \lambda_{1}\right)$, with $\lambda_{1}(x, a)=\alpha a x^{-\rho}(\alpha>0)$, and $F_{2} \in D_{d}\left(m, \lambda_{2}\right)$, with $\lambda_{2}(x, a)=\beta a x^{-\rho}(\beta>0)$. We determine whether $F \in D_{d}\left(m, \lambda_{3}\right)$ for some function $\lambda_{3}$.

Start by considering

$$
F(t x+a, t y+b)-F(t x, t y)=\Psi\left(F_{1}(t x+a), F_{2}(t y+b)\right)-\Psi\left(F_{1}(t x), F_{2}(t y)\right) .
$$

Assume that $\psi$ has continuous derivatives of order 2. Using Taylor's Theorem with remainder, there exists $z=\left(z_{1}, z_{2}\right)$ with $0 \leqslant z_{1} \leqslant x$ and $0 \leqslant z_{2} \leqslant y$ such that

$$
\begin{aligned}
& \Psi(x+a, y+b)-\Psi(x, y) \\
& \quad=a \Psi_{1}(x, y)+b \Psi_{2}(x, y)+\frac{a^{2}}{2} \Psi_{1,1}\left(z_{1}, z_{2}\right)+\frac{b^{2}}{2} \Psi_{2,2}\left(z_{1}, z_{2}\right)+a b \Psi_{1,2}\left(z_{1}, z_{2}\right),
\end{aligned}
$$

where $\Psi_{i}$ and $\Psi_{i, j}$ are the first and second partial derivatives of $\Psi$. If the derivatives of order 2 are bounded, then

$$
\Psi(x+a, y+b)-\Psi(x, y)=a \Psi_{1}(x, y)+b \Psi_{2}(x, y)+O(1)\left(a^{2}+b^{2}+a b\right)
$$

which gives

$$
\begin{aligned}
\Psi\left(F_{1}(t x+\right. & \left.a), F_{2}(t y+b)\right)-\Psi\left(F_{1}(t x), F_{2}(t y)\right) \\
= & {\left[F_{1}(t x+a)-F_{1}(t x)\right] \Psi_{1}\left(F_{1}(t x), F_{2}(t y)\right) } \\
& +\left[F_{2}(t y+b)-F_{2}(t y)\right] \Psi_{2}\left(F_{1}(t x), F_{2}(t y)\right) \\
& +O(1)\left[F_{1}(t x+a)-F_{1}(t x)\right]^{2}+O(1)\left[F_{2}(t y+b)-F_{2}(t y)\right]^{2} \\
& +O(1)\left[F_{1}(t x+a)-F_{1}(t x)\right]\left[F_{2}(t y+b)-F_{2}(t y)\right] .
\end{aligned}
$$

This gives

$$
\begin{aligned}
& \frac{\Psi\left(F_{1}(t x+a), F_{2}(t y+b)\right)-\Psi\left(F_{1}(t x), F_{2}(t y)\right)}{m(t)} \\
& \sim \alpha a x^{\rho} \Psi_{1}\left(F_{1}(t x), F_{2}(t y)\right) \beta b y^{\rho} \Psi_{2}\left(F_{1}(t x), F_{2}(t y)\right)+O(1) m(t),
\end{aligned}
$$

for some $\alpha, \beta$.

From Omey (1994, p. 113) we have that $m(t)=O\left(\bar{F}_{1}(t)\right)$ and $m(t)=O\left(\bar{F}_{2}(t)\right)$. Hence, 


$$
\begin{aligned}
& \frac{\Psi\left(F_{1}(t x+a), F_{2}(t y+b)\right)-\Psi\left(F_{1}(t x), F_{2}(t y)\right)}{m(t)} \\
& \sim \alpha a x^{\rho} \Psi_{1}\left(F_{1}(t x), F_{2}(t y)\right)+\beta b y^{\rho} \Psi_{2}\left(F_{1}(t x), F_{2}(t y)\right) .
\end{aligned}
$$

As a specific example, suppose we define $\Psi(x, y)$ by an Archimedian copula (see, for example, Balakrishnan and Lai (2009), p. 37) that is defined by a continuous, decreasing generator function $\phi$ from $[0,1]$ to $[0, \infty)$ such that $\phi(\Psi(x, y))=\phi(x)+$ $\phi(y)$.

$\Psi$ is a copula if and only if its pseudoinverse, given by

$$
\phi^{[-1]}= \begin{cases}\phi^{-1}(t), & 0 \leqslant t \leqslant \phi(0) \\ 0, & \phi(0) \leqslant t \leqslant \infty,\end{cases}
$$

is decreasing and convex, in which case, $\Psi(x, y)=\phi^{-1}(\phi(x)+\phi(y))$.

The complementary copula is defined by

$$
\hat{\Psi}(x, y)=x+y-1+\Psi(1-x, 1-y),
$$

so that

$$
\Psi(x, y)=x+y-1+\hat{\Psi}(1-x, 1-y) .
$$

A particular type of Archimedian copula is the bivariate Pareto copula for which $\phi(t)=t^{-1 / \delta}-1, \delta>0$. This gives $\hat{\Psi}(x, y)=\left(x^{-1 / \delta}+y^{-1 / \delta}-1\right)^{-\delta}$ and

$$
\Psi(x, y)=x+y-1+\left((1-x)^{-1 / \delta}+(1-y)^{-1 / \delta}-1\right)^{-\delta} .
$$

Differentiating $\Psi$ gives

$$
\begin{aligned}
\Psi_{1}(x, y) & =1-\left[(1-x)^{-1 / \delta}+(1-y)^{-1 / \delta}-1\right]^{-\delta-1}(1-x)^{-1-1 / \delta} \\
& =1-\left[(1-x)^{1 / \delta}\left((1-x)^{-1 / \delta}+(1-y)^{-1 / \delta}-1\right)\right]^{-\delta-1} \\
& =1-\left[1+\left(\frac{1-x}{1-y}\right)^{1 / \delta}-(1-x)^{1 / \delta}\right]^{-\delta-1} .
\end{aligned}
$$

Substituting $F_{1}$ and $F_{2}$ gives

$$
\Psi_{1}\left(F_{1}(t x), F_{2}(t y)\right)=1-\left[1+\left(\frac{\bar{F}_{1}(t x)}{\bar{F}_{2}(t y)}\right)^{1 / \delta}-\bar{F}_{1}(x)^{1 / \delta}\right]^{-\delta-1} .
$$

If $\alpha>\beta$, then $\bar{F}_{1}(t x)=o\left(\bar{F}_{2}(t y)\right)$ and $\Psi_{1}\left(F_{1}(t x), F_{2}(t y)\right) \rightarrow 0$.

If $\alpha<\beta$, then, $\bar{F}_{1}(x) / \bar{F}_{2}(x) \in R V_{-\alpha+\beta}$ and

$$
\Psi_{1}\left(F_{1}(t x), F_{2}(t y)\right) \sim 1-(x / y)^{(-\alpha+\beta)(-\delta-1) / \delta} \rightarrow 1 .
$$

If $\bar{F}_{1} \sim \bar{F}_{2} \in R V_{-\alpha}$, so that $\alpha=\beta$, then

$$
\Psi_{1}\left(F_{1}(t x), F_{2}(t y)\right) \rightarrow 1-\left[1+(x / y)^{-\alpha / \delta}\right]^{-\delta-1} .
$$

After deriving similar results for $\Psi_{2}\left(F_{1}(t x), F_{2}(t y)\right)$ we can obtain expressions for (3.3). 


\section{Concluding remarks}

(1) To obtain inequalities, we only need detailed information about the marginal distributions. In order to obtain asymptotic equalities, we have to assume that in each of the tails we can use the same auxiliary regularly varying function $m(t)$. We can relax this assumption by looking at the following class of d.f.. For simplicity, we only give the definition in $\mathbb{R}^{2}$.

Definition 4. We say that the d.f. $F$ is in the class $D_{d}(m, \vec{c}, \lambda)$ if we have $m(t), c_{1}(t), c_{2}(t) \in R V$ and if

$$
\frac{F\left(c_{1}(t) x_{1}, c_{2}(t) x_{2}\right)-F\left(c_{1}(t) x_{1}-a_{1}, c_{2}(t) x_{2}-a_{2}\right)}{m(t)} \rightarrow \lambda(\vec{x}, \vec{a}),
$$

for all $\vec{x}>\overrightarrow{0}$ with $x^{0}<\infty$ and $\vec{a} \in \mathbb{R}^{2}$.

(2) It is not clear under what general conditions $F \in D_{d}(m, \lambda)$ implies that the defining property holds locally uniformly in $\vec{x}$.

(3) In our future research, we will discuss properties of the classes defined below. In each case, we consider limits as $x^{0} \rightarrow \infty$.

$$
\begin{array}{ll}
f \in O D(m): & \forall \vec{y},|f(\vec{x}+\vec{y})-f(\vec{x})|=O(1) m(\vec{x}) ; \\
f \in D(m, \lambda): & \forall \vec{y},(f(\vec{x}+\vec{y})-f(\vec{x})) / m(\vec{x}) \rightarrow \lambda(\vec{y}) ; \\
f \in L(\lambda): & \forall \vec{y}, f(\vec{x}+\vec{y}) / f(\vec{x}) \rightarrow \lambda(\vec{y})
\end{array}
$$

and, so on.

Suppose, for example, that $F$ is a d.f.with marginals $F_{i} \in O D\left(m_{i}\right)$. Using the inequality $|F(\vec{x}+\vec{y})-F(\vec{x})| \leqslant \sum_{i=1}^{d}\left|F_{i}\left(x_{i}+y_{i}\right)-F_{i}\left(x_{i}\right)\right|$ and $F_{i} \in O D\left(m_{i}\right)$, we obtain that $|F(\vec{x}+\vec{y})-F(\vec{x})|=O(1) \sum_{i=1}^{d} m_{i}\left(x_{i}\right)$, as $x^{0} \rightarrow \infty$. It follows that $F \in O D(m)$ with $m(\vec{x})=\sum_{i=1}^{d} m_{i}\left(x_{i}\right)$. Note that if $m_{i}(x) \in O R V$, then as $x^{0} \rightarrow \infty$

$$
m\left(x_{1} y_{1}, \ldots, x_{d} y_{d}\right)=\sum_{i=1}^{d} m_{i}\left(x_{i} y_{i}\right)=O(1) \sum_{i=1}^{d} m_{i}\left(x_{i}\right)=O(1) m(\vec{x}) .
$$

(4) In Corollary 3.5, we provided conditions under which

$$
\frac{1}{m(t)}\left(1-F^{* n}(t \vec{x}-\vec{a})-n(1-F(t \vec{x}-\vec{a}))\right) \rightarrow \Psi_{n}(\vec{x}, \vec{a}) .
$$

Taking $\vec{a}=\overrightarrow{0}$, we have the following probabilistic interpretation. We have

$$
\begin{aligned}
1-F^{* n}(t \vec{x}) & =P\left(\max _{i}\left(S_{n, i} / x_{i}\right)>t\right), \\
1-F(t \vec{x}) & =P\left(\max _{i}\left(X_{i} / x_{i}\right)>t\right) .
\end{aligned}
$$

It follows that

$$
P\left(\max _{i}\left(S_{n, i} / x_{i}\right)>t\right) \sim n P\left(\max _{i}\left(X_{i} / x_{i}\right)>t\right)
$$

and that

$$
P\left(\max _{i}\left(S_{n, i} / x_{i}\right)>t\right)-n P\left(\max _{i}\left(X_{i} / x_{i}\right)>t\right) \sim m(t) \Psi_{n}(\vec{x}, \overrightarrow{0}) .
$$




\section{References}

1. N. Balakrishnan and C.-D. Lai, Continuous Bivariate Distributions, Second ed., Springer, Dordrecht, 2009

2. A. Baltrunas and E. Omey, The rate of convergence for subexponential distributions, Liet. Mat. Rink. 38(1) (1998), 1-18.

3. A. Baltrunas, E. Omey and S. Van Gulck, Hazard rates and subexponential distributions, Publ. Inst. Math., Nouv. Sér. 80(94), (2006), 29-46

4. N.H. Bingham, C.M. Goldie and J.L. Teugels, Regular Variation, Cambridge University Press, 1987.

5. D. J. Daley, E. Omey and R. Vesilo, The tail behaviour of a random sum of subexponential random variables and vectors Extremes 10 (2007), 21-39.

6. J. L. Geluk and L. de Haan, Regular Variation, Extensions and Tauberian Theorems, CWI Tract 40, Centre for Mathematics and Computer Science, Amsterdam, The Netherlands, 1987

7. J. Geluk and A. G. Pakes, Second-order subexponential distributions, J. Aust. Math. Soc., Ser. A 51 (1991), 73-87.

8. E. Omey, Multivariate Regular Variation and Its Applications in Probability Theory, Ph.D. Thesis, Catholic University of Leuven (Belgium), 1982 (In Dutch).

9. E. Omey, On the difference between the product and the convolution product of distribution functions, Publ. Inst. Math., Nouv. Sér. 55(69) (1994), 111-145.

10. E. Omey, On a subclass of regularly varying functions, J. Statist. Plann. Inference 45 (1995), $275-290$.

11. E. Omey, Subexponential distributions and the difference between the product and the convolution product of distribution functions in $\mathbb{R}^{d}$, J. Math. Sci. (N.Y.) 138 (2006), 5434-5449.

12. E. Omey, F. Mallor and J. Santos, Multivariate subexponential distributions and random sums of random vectors, Adv. Appl. Probab. 38 (2006), 1028-1046.

13. E. Seneta, Regularly Varying Functions Lecture Notes 508, Springer, Berlin, 1976.

14. A. Yakymiv, Multivariate Tauberian theorems and their application to Bellman-Harris branching processes, Math. USSR Sbornik 115(3) (1981), 463-477.

EHSAL-HUB

(Received 1103 2010)

Stormstraat 2

(Revised 0202 2011)

1000 Brussels, Belgium.

edward.omey@hubrussel . be

Department of Electronic Engineering

Macquarie University, NSW, 2109

Australia

rein.vesilo@mq.edu.au 